1994-07-01

\title{
Emitted Current Instability from Silicon Field Emission Emitters Due to Sputtering by Residual Gas Ions
}

W.I. Karain

Larry V. Knight

larry_knight@byu.edu

David D. Allred

allred@byu.edu

A. Reyes-Mena

Follow this and additional works at: https://scholarsarchive.byu.edu/facpub

Part of the Astrophysics and Astronomy Commons, and the Physics Commons

\section{Original Publication Citation}

The following article appeared in W.I. Karain, L. V. Knight, D. D. Allred and A. Reyes-Mena, "Emitted current instability from silicon field emission emitters due to sputtering by residual gas ions," Journal of Vacuum Science Technology A 12(4), 258185 (1994). and can be found at [http://avspublications.org/jvsta/resource/1/jvtad6/v12/i4/p2581_s1][http://dx.doi.org/1.1116/ 1.57961].

\section{BYU ScholarsArchive Citation}

Karain, W.I.; Knight, Larry V.; Allred, David D.; and Reyes-Mena, A., "Emitted Current Instability from Silicon Field Emission Emitters Due to Sputtering by Residual Gas Ions" (1994). Faculty Publications. 1169. https://scholarsarchive.byu.edu/facpub/1169 accepted for inclusion in Faculty Publications by an authorized administrator of BYU ScholarsArchive. For more information, please contact ellen_amatangelo@byu.edu. 


\title{
Emitted current instability from silicon field emission emitters due to sputtering by residual gas ions
}

\author{
W. I. Karain, Larry V. Knight, David. D. Allred, and A. Reyes-Mena ${ }^{a), b\}}$ \\ Department of Physics and Astronomy, Brigham Young Universiry, Provo, Utah 84602
}

(Received 15 November 1993; accepted 11 April 1994)

\begin{abstract}
We have fabricated arrays of silicon field emitters using semiconductor lithography techniques. The density of the tips was $10 \% \mathrm{~cm}^{2}$. The maximum current that can be extracted from each emitter is limited by resistive heating. We have investigated how the electron current emitted changes under constant applied voltage. We found that the current is very sensitive to the vacuum conditions. We attribute this to sputtering of the emitters due to ionized residual gas molecules. The poorer the vacuum, the higher the instability in the current. We studied this phenomenon at $10^{6}$ and $10^{-8}$ Tor. The model of two concentric spherical shells is used to obtain the ion energy distribution. This is then used to calculate the rate of ion bombardment and the rate of atoms sputtered. A lifetime of the tip can be deduced from these calculations.
\end{abstract}

\section{INTRODUCTION}

There is considerable interest in soft $\mathrm{x}$-ray sources which can be modulated temporally and spatially. Such a source has been developed by Stearns at LLNL and is called the $\mathrm{x}$-ray diode. ${ }^{1}$ It consists of a photocathode spaced a few millimeters of vacuum from a thin $x$-ray anode. The photoelectrons emitted from the photocathode are accelerated toward the anode where they produce $x$ rays upon collision. Duc to the proximity of the photocathode to the anode, the x-ray output can be modulated temporally and spatially by modulating the light incident on the photocathode. A limitation of this design is that the photocathode needs a ultrahigh vacuum atmosphere for stable emission. Recently we have reported the fabrication of silicon field emission arrays. ${ }^{2-4}$ in this article we studied the stability of the emission current under various vacuum conditions. We found that the poorer the vacuum, the higher the instability in the current. The model of two concentric spherical shells $s^{5}$ is used to estimate the rate of ion bombardment and the rate of atoms sputtered.

\section{EXPERIMENT}

Large areas of silicon tips were fabricated on $p$-type single crystal silicon wafers using standard lithography techniques. ${ }^{1,2}$ After etching, the wafer was washed in deionized water, methanol and dried in nitrogen. The density of the tips was $10^{5} / \mathrm{cm}^{2}$. A typical radius tip was $10 \mathrm{~nm}$. The wafers were diced to facilitate testing. Each die consisted of 16000 tips and has an area of $16 \mathrm{~mm}^{2}$. The die was then glued to an aluminum piate using colloidal silver. Using a micrometer, prior to closing and evacuating the vacuum chamber, this plate was positioned within a hundred microns of a transparent conductive glass plate which serves as the collecting anode. Emission testing in an ungated configuration was done at various pressures. A mechanical pump in

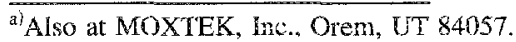

b) On leave from the Programa Multidiciplinario de Materiales Avanzados, Centro de Investigación y de Estudios Avanzados del Instituto Politécnico Nacional, Unidad Saitillo, 25000 Saltillo, Coahuila, México.
}

conjunction with a turbo molecular pump were used to evacuate the unbaked chamber. The pressure was monitored with an ion gauge tube, and a mass spectrum analyzer was used to examine the residual gas content in the system. Emission currents were measured with the ion gauge filament on and off and no differences in the readings were observed. The current was measured as a voltage drop across a known resistor using a Keithley ammeter which has a $10 \mathrm{G} \Omega$ internal impedance.

\section{THEORETICAL BACKGROUND: RESULTS AND DISCUSSION}

\section{A. Resistive heating damage}

The emitter surface temperature is given by the following equation: $:^{6,7}$

$$
T_{s}=T_{0} J^{2} \rho m^{2} / 2 \kappa,
$$

where $T_{s}$ is the tip surface temperature, $\rho$ is the electric resistivity, $\kappa$ is the thermal conductivity, and $T_{0}$ is the substrate temperature taken here to be room temperature. $m$ is related to the tip radius through

$$
r=m \nu_{0},
$$

where $r$ is the tip radius, and $y_{0}$ is the tip half-angle. ${ }^{5}$

The thermal conductivity of silicon decreases as temperature increases. The melting temperature of silicon is $1690 \mathrm{~K}$. Both $\kappa$ and $\rho$ vary with temperature. According to Doian, the use of values for intermediate temperatures is satisfactory. The thermal conductivity value at $1000 \mathrm{~K}$ of 0.4 W/cm K will be used. ${ }^{3}$ The resistivity of a $p$-type silicon wafer increases from $10 \Omega \mathrm{cm}$ at room temperature to 40 $\Omega \mathrm{cm}$ at $150^{\circ} \mathrm{C}$, then decreases. For the worst case scenario of maximum temperature increase, this value of $40 \Omega \mathrm{cm}$ will be used.

For a typical tip with a radius of $100 \AA$, and $\nu_{0}=10^{\circ}$, the tip will be at the melting temperature if the emitted current density is larger than $0.92 \times 10^{6} \mathrm{~A} / \mathrm{cm}^{2}$ according to Eq. (1). Melting of tips has been observed (Fig. 1). 


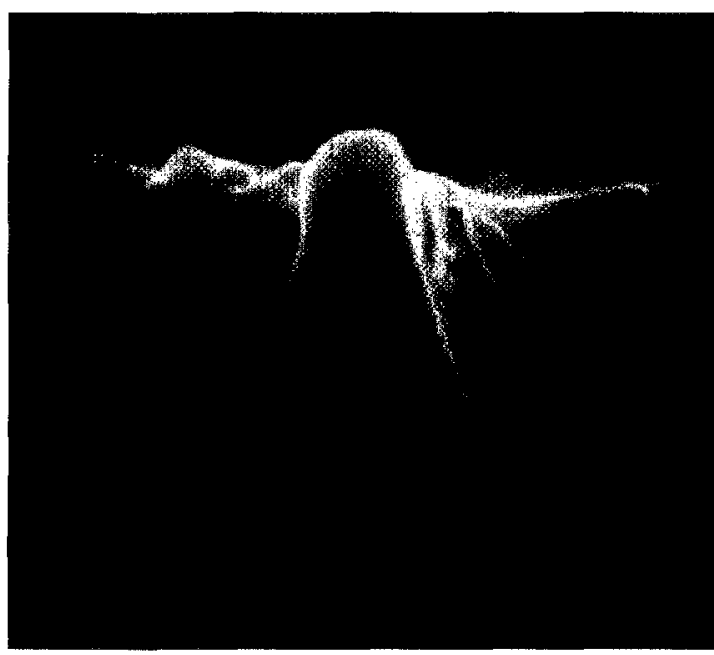

FIG. 1. Melting of tips.

For a $100 \AA$ tip, the emitting area is given by ${ }^{6}$

$A=2 \pi m^{2}\left(1-\cos \nu_{0}\right)$.

Thus to attain the required current densities to melt the tip, the current would have to be around 3 microamps. However, this is close to a typical total current measured from the whole array. The likelihood of the current coming from one tip is very small. We detected emission from more than one tip. This was verified visually. Figure 2 shows a FowlerNordheim plot of the current emission at room temperature for the array in the dark. At low voltages (region I), the log of the emitted current is linear with the reciprocal voltage. The electron supply is adequate for the emission to be limited only by the barrier transparency. As the voltage increases, the current begins to saturate (region II). Total emission currents (due to approximately 16000 tips) were as high as 16 microamperes. The emitted current caused fluorescence of the conductive tin oxide glass anode. Based on these emis-

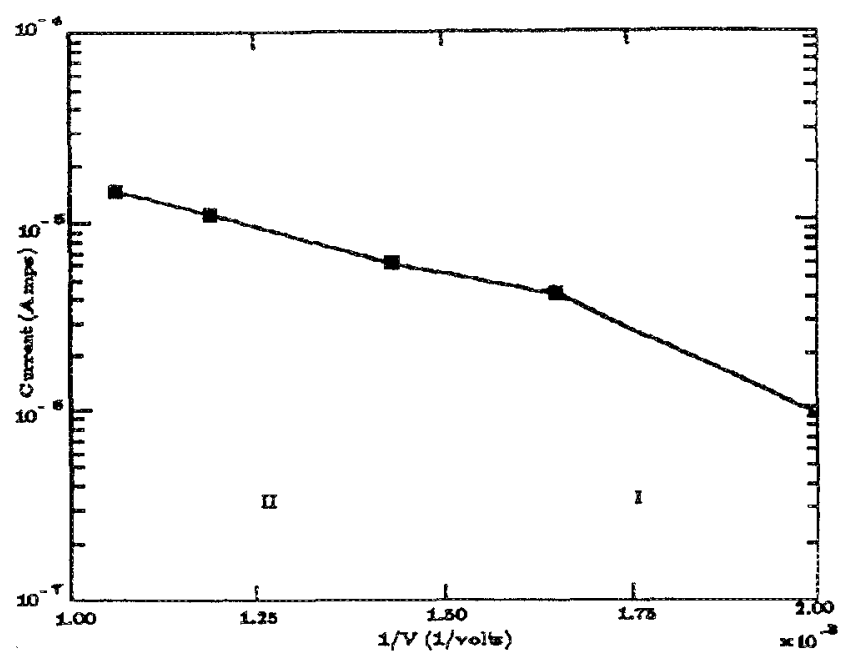

FIG. 2. Experimental graph of the emitted current vs voltage.

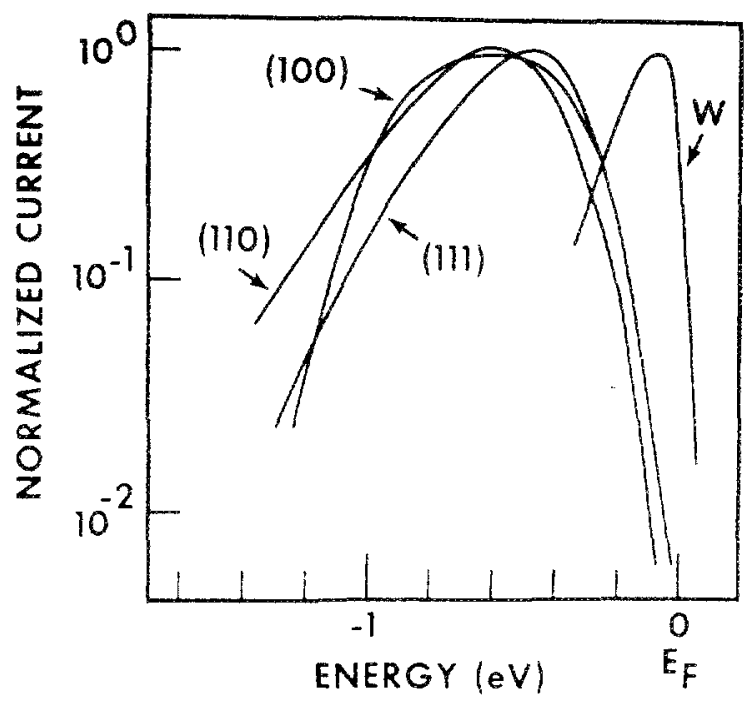

FIG. 3. Electron energy distribution from $p$-type silicon (Ref. 11).

sion current values, the current from each tip was on the order of several nanoamperes. Thus to realize the current densities required to cause damage, the emission would have to come from a very small area. This emission would basically come from atomic scale protrusions on the tip. Spind et al. report that field emitters not treated with field/heat methods give "low emission areas." A protrusion with a radius of $10 \AA$ would decrease the emitting area by a factor of 100 . A current of about $30 \mathrm{nA}$ would be enough to cause the protrusion to melt. This local increase in temperature and gas pressure could lead to an unstable situation where the whole tip is destroyed by a local arc. This would explain the damage detected with the scanning electron microscope.

\section{B. Nottingham effect}

The Nottingham effect tries to hold the emitting surface at a temperature given by ${ }^{10}$

$$
T_{c}=5.32 \times 10^{-5} E / \phi^{1 / 2},
$$

where $E$ is the electric field applied to the surface, and $\phi$ is the work function. This is the equilibrium temperature brought about by a balance between the energy given up to the tip by electrons emitted below the Fermi level, and the energy removed from the tip by electrons emitted above the Fermi level. To emit a current density of $\approx 1 \times 10^{6} \mathrm{~A} / \mathrm{cm}^{2}$, the electric field needed is calculated using the FowlerNordheim equation and is $\approx 5.6 \times 10^{7} \mathrm{~V} / \mathrm{cm}$. Then $T_{c} \approx 1400$ $K$. This is lower than the melting temperature. Thus considerable heating of the tip occurs due to this phenomena. However, it appears that this mechanism is not capable of stabilizing the temperature below the melting point. This conclusion is arrived at because damage of tips has been observed with a scanning electron microscope.

Using a different approach to look at the Nottingham effect, we will use the energy distribution of electrons emitted from silicon tips ${ }^{11}$ (Fig. 3). Lewis et al. found that electrons emitted at room temperature originated below the Fermi 
level. If each emitted electron has $0.4 \mathrm{eV}$ of energy, with each tip emitting $1 \mathrm{nA}$, the amount of power given to the tip is $4 \times 10^{-10} \mathrm{~J} / \mathrm{s}$. Using the heat conduction law, the temperature change for the tip is

$$
\Delta T=P l / \kappa A,
$$

where $P$ is the heat given up to the tip by the emitted electrons, $l$ is the tip length (the tip is assumed to be a cylinder), $\kappa$ is the silicon thermal conductivity, and $A$ is the crosssectional area. Assuming the tip has a radius of $100 \AA$, and a length of $10 \mu \mathrm{m}$, the temperature change is $1 \mathrm{~K}$. However, if the emitted current is $1.7 \mu \mathrm{A}$, the temperature change is $1700 \mathrm{~K}$, enough to melt the tip. This is close to the value obtained from the resistive heating calculation $(3 \mu \mathrm{A})$. This value would be the maximum current that can be emitted from the whole tip, not protrusions, before damage occurs due to resistive heating and the Nottingham effect. In this saturation region the electric field penetration into the semiconductor is not strong enough to cause impact multiplication of carriers or any avalanching effects of electrons.

\section{Ion sputtering}

The number of ions hitting the emitting surface is ${ }^{5}$

$$
n=\int_{r_{i}}^{r_{a}} \frac{I_{e}}{e} N Q\left(V_{r}\right) d r
$$

where $I_{e}$ is the emitted current, $N=$ pressure $\times 3.55 \times 10^{16}$ molecules $/ \mathrm{cm}^{3}, Q\left(V_{r}\right)$ is the residual gas ionization cross section. The number of atoms sputtered is ${ }^{5}$

$$
n_{s}=\int_{r_{s}}^{r_{m}} \frac{I_{e}}{e} N Q\left(V_{r}\right) Y\left(V_{r}\right) d r
$$

where $Y$ is the sputtering yield, $r_{i}=$ radius at which energy of electrons reaches the gas ionization threshold, $r_{s}=$ radius of ion threshold for sputtering, $r_{m}=$ maximum radius for an ion to hit the emitter surface.

The potential distribution, $V_{r}$ to be used here is that of two concentric shells 5 and is given by

$$
V_{r}=V_{A}\left(r_{0} / r-1\right)\left(r_{0} / r_{A}-1\right)^{-1} \text {, }
$$

which can be simplified to

$$
V_{r} \approx-V_{A}\left(r_{0} / r-1\right)
$$

for $r_{0} \ll r_{A} . V_{r}$ is the voltage at radius $r, V_{A}$ is the voltage applied between the anode and cathode, $r_{A}$ is the cathodeanode separation, and $r_{0}$ is the emitter tip radius.

For this voltage distribution, the radi $r_{i}$ and $r_{s}$ are very close to $r_{0} . r_{m}$ is given by ${ }^{5}$

$$
r_{m}=r_{0}(a / 0.63)\left(V_{A} / V_{\text {th }}\right)^{1 / 3} \text {, }
$$

with

$$
0.4 \leqslant a \leqslant 0.7
$$

assuming that each molecule has an initial energy $V_{\text {th }}$ in the transverse direction due to thermal energy.

The ionization cross section and the sputtering yield are both energy dependent. There is no analytic form for either of these variables. An average value of $Q$ and $Y$ will be substituted at the average potential $\bar{V}$ between $V_{A}$ and $V_{r}$ where $V$ is given by

$$
\bar{V}=1 /\left(r_{m}-r_{0}\right) \int_{r_{0}}^{r_{a}} V_{A}\left(\frac{r_{0}}{r}-1\right) d r .
$$

Therefore, the number of ions hitting the emitting surface is

$$
n=\left(\frac{I_{e}}{e}\right) N Q(\bar{V})\left(r_{m}-r_{0}\right),
$$

and the number of atoms sputtered is

$$
n_{s}=\left(\frac{I_{e}}{e}\right) N Q(\bar{V}) Y(\bar{V})\left(r_{m}-r_{0}\right) .
$$

A mass spectrum analyzer was used to examine the residual gas content in the $10^{-6}$ Torr system. Water vapor molecules, carbon monoxide and nitrogen, and oxygen were the top three gases in that order. Thus it is likely that the ion species responsible for sputtering will be hydrogen, oxygen, and nitrogen. The sputtering yield of hydrogen is about two orders of magnitude less than the other two, so we ignore its contribution. As for oxygen and nitrogen, their atomic masses are close, and thus we will assume, for the lack of published data, that they have the same sputtering yield. It is an established fact that the sputtering yields for ions of nearly equal mass is the same at low ion energies. ${ }^{12}$ In Fig. 4, there is one data point for nitrogen at $\approx 50 \mathrm{keV}^{13}$ By assuming that nitrogen and neon (which has abundant yield data) behave the same at low energies, we estimate that the sputtering yield of silicon by nitrogen at $1 \mathrm{keV}$ is $\approx 0.4$, and $\approx 0.45$ at $2 \mathrm{keV}$ (Fig. 4). The ionization cross section will be taken to be $\approx 10^{-16} \mathrm{~cm}^{2}$ because values above $700 \mathrm{~V}$ could not be found.

\section{Lifetime versus sputtering}

The following data was collected in a $10^{-6}$ and $10^{-8}$ Tor system. The analysis will be based on the model developed above, and it will try to explain the effect of the vacuum environment on emitter lifetime and stability.

We propose a mechanism where the tips providing the current melted due to resistive heating brought about by increased roughness due to bombardment by ions.

For the cycle to be analyzed: $I_{e}=10^{-9} \mathrm{~A}, P=10^{-6}$ Torr, $V_{A}=V_{\mathrm{th}}=1800 \mathrm{~V}$ (for $\left.a \approx 0.6\right), r_{0}=100 \AA, r_{m}=42 r_{0}$, $\vec{V}=1636 \mathrm{~V}, Q=1 \times 10^{-16} \mathrm{~cm}^{2}$, and $Y \approx 0.45$. Using Eq. (13), the atom sputtering rate is: $n_{s}=0.41$ atoms $/ \mathrm{s}$.

The number of silicon atoms forming one layer at the surface of the emitter is estimated by dividing the surface area of the emitter, by that of one silicon atom. For a tip with a radius of $100 \AA$, there are $\approx 340$ silicon atoms in one monolayer. Using the value for $n_{s}$ calculated above, the time to remove one monolayer of silicon would be $\approx 14 \mathrm{~min}$. It has to be kept in mind that the radii of the tips range from values lower than $100 \AA$, to values as large as a few microns. Thus, the time to sputter one monolayer will actually be 


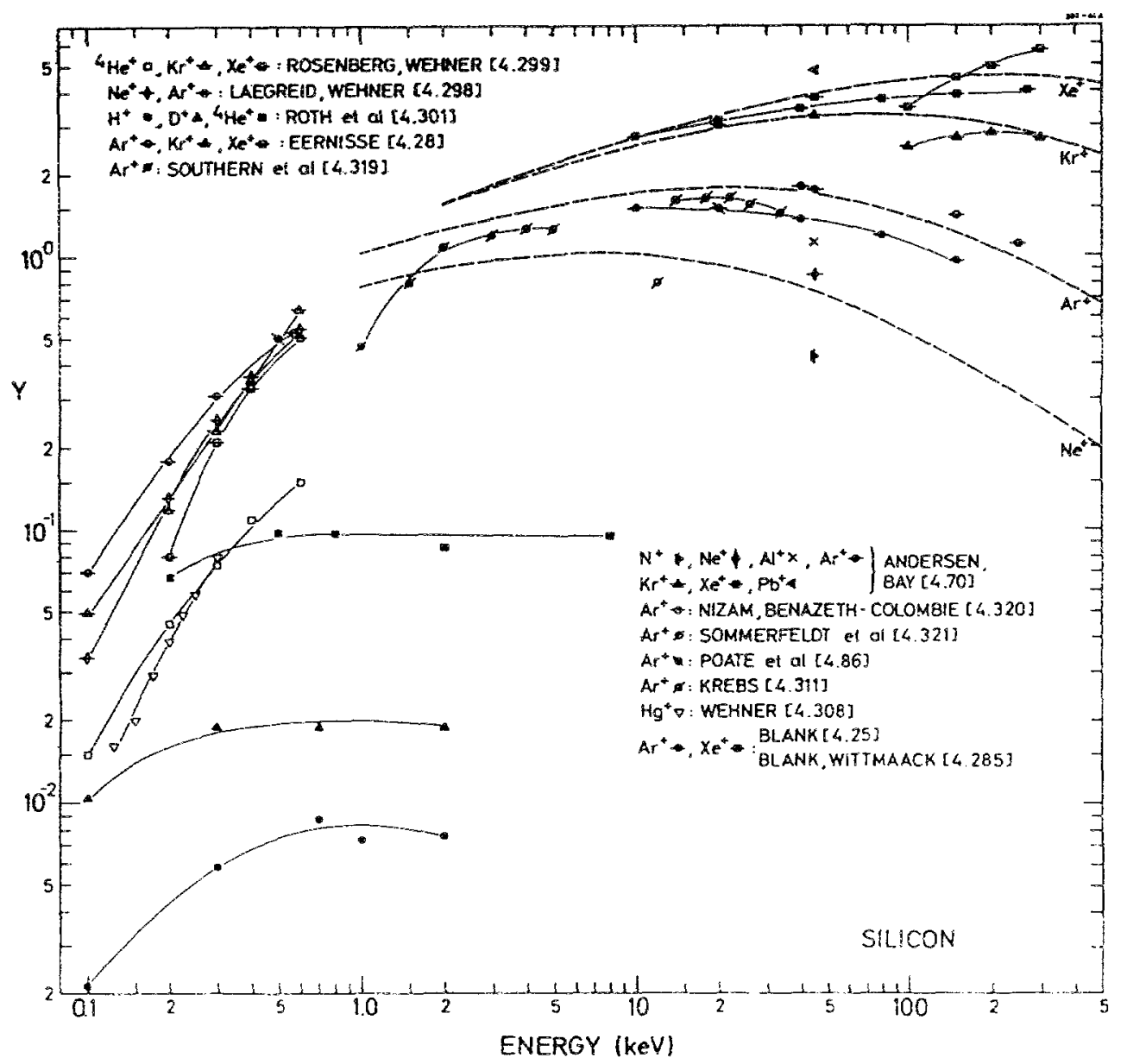

FIG. 4. Silicon sputter yield (Ref. 13).

different for different tips. However, even with a monolayer sputtering time of several hours for the thicker tips, the lifetime is not satisfactory for practical use.

The whole cycle is compressed into Fig. 5 . It began at $\approx 1$ $\mu \mathrm{A}$. It steadily increased to $\approx 2 \mu \mathrm{A}$ after $5.7 \mathrm{~h}$. Then over a period of $2.5 \mathrm{~h}$, the current increased from $\approx 2$ to $\approx 3 \mu \mathrm{A}$, and noise increased significantly. It was steady for $55 \mathrm{~min}$,

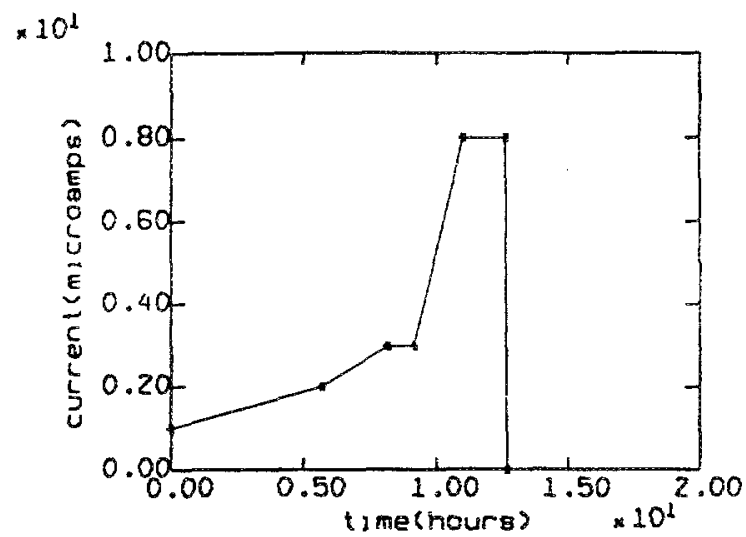

Fig. 5. Current vs time at constant voitage at $10^{-6}$ Torr. then it started to increase steadily, and noise increased. After a total of $12.6 \mathrm{~h}$ the current dropped from $\approx 8 \mu \mathrm{A}$ to less than $0.1 \mu \mathrm{A}$.

It appears that the current increases steadily, with relatively low noise for several hours. This is followed by a significant increase in the noise, and a sudden drop in the current. This is probably when damage occurs via arcing.

The sputtering rate is very high. While some tips will be blunted, and effectively put out of use, the sputtering of others appear to sharpen them up. Initially, this probably leads to the "clean" steady increase in the current. As the surface roughness increases, the increase in the local field intensification factor, $\beta$, leads to a large local increase in the emitted current density. This leads to tip melting via resistive heating. This will explain the sudden drop in the current as being due to the damage of the emitting tips.

Martin et $a l^{14}$ observed a similar effect for tungsten field emitters. They attributed the increase in the current to an increase in $\beta$ due to sputtering by helium ions in the cavity. They noted that when the current changed by about a factor of five, the current voltage characteristics became erratic, and cathode damage was "likely."

To compare, the emitted current in a $10^{-8}$ Torr system was measured over a period of several days. The cycle is compressed into Fig. 6. 


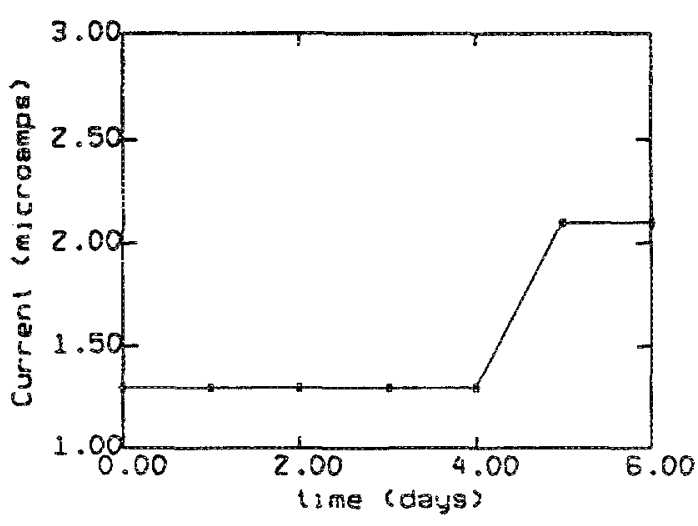

Frg. 6. Current vs time at constant voltage at $10^{-8}$ Torr.

Even though the average current stayed close to the initial value, the noise increased. The current emitted from each tip was $\approx 10^{-10} \mathrm{~A}$. The applied voltage was $1200 \mathrm{~V}$. By using the formulas above, it was found that $716 \mathrm{~h}$ are needed to remove one monolayer. Obviously, a better vacuum means longer lifetime.

\section{CONCLUSIONS}

We have investigated the stability of the emission current extracted from arrays of silicon field emitters under constant applied voltage at $10^{-6}$ and $10^{-8}$ Torr. We found that the poorer the vacuum the higher the instability in the current. Based on the model of two concentric spherical shells we propose a mechanism where the tips providing the current melted due to resistive heating brought about by increased roughness due to sputtering by residual gas ions. Also, lifetimes of the tips were deduced from these calculations.

${ }^{1}$ D. G. Stearns, Nucl. Instrum. Methods Phys. Res. A 242, 364 (1986).

${ }^{2}$ W. I. Karain, L. V. Knight, D. D. Allred, and A. Reyes-Mena, Proc, SPIE 1741, 12 (1993).

${ }^{3}$ W. I. Karain, I. V. Knight, D. Allred, and A. Reyes-Mena, Encylia (to be published).

${ }^{4}$ W. I. Karain, L. V. Knight, D. Allred, and A. Reyes-Mena, J. Nanostruct. Mater. (to be published).

${ }^{5}$ I. Brodie, Int. J. Electron. 38, 541 (1975).

${ }^{6}$ W. W. Dolan, W. P. Dyke, and J. K. Trolan, Phys. Rev. 91, 1054 (1953).

${ }^{7}$ A. I. Chapman, Fundamentals of Heat Transfer (Macmillian, New York, 1987), Chap. 1.

${ }^{8}$ H. F. Wolf, Silicon Semiconductor Data (Pergamon, New York, 1969).

${ }^{9}$ C. A. Spindt, I. Brodie, L. Humphrey, and E. R. Westerburg, I. Appl. Phys. 47, 5248 (1976).

${ }^{10}$ F. M. Charbonnier, R. W. Strayer, L. W. Swanson, and E. E. Martin, Phys. Rev. Lett. 13, 397 (1964).

${ }^{11}$ B. F. Lewis and T. E. Fisher, Surf. Sci. 41, 371 (1974).

${ }^{12}$ R. V. Stuart and G. K. Wehner, J. Appl. Phys. 33, 2345 (1962).

${ }^{13}$ Sputtering by Particle Bombardment $I$, edited by R. Behrisch (Springer, Berlin, 1981), p. 169.

${ }^{14}$ E. E. Martin, J. K. Trolan, and W. P. Dyke, J. Appl. Phys. 31, 782 (1983). 